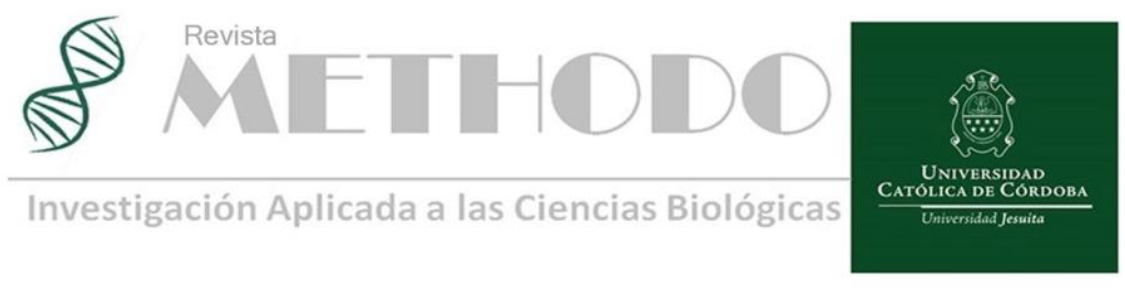

\title{
Utilidad de los factores pronósticos para la detección de lesiones pre-neoplásicas de colon
}

\section{Utility of prognostic factors in the detection of pre-neoplastic colon lesions}

\author{
Marianela P. Bedini ${ }^{1}$, Juan José Trakál ${ }^{1}$, Esteban Trakál ${ }^{1}$ \\ 1 Universidad Católica de Córdoba. Clínica Universitaria Reina Fabiola. Servicio de Gastroenterología \\ Correspondencia: Marianela Bedini. Servicio de Gastroenterología. Clínica Universitaria Reina Fabiola Oncativo 1248-X5004FHP-Cordoba, Argentina; email: \\ marianelabedini@gmail.com
}

\section{Resumen}

INTRODUCCIÓN: El cáncer colorrectal (CCR) representa un problema de salud pública mundial. El objetivo de la participación de los pacientes en programas de screening de cáncer colorrectal (SCCR) es realizar undiagnóstico temprano y reducir la mortalidad.

Los estudios endoscópicos son una herramienta clave para estratificar el riesgo y establecer el seguimiento de los pacientes.

OBJETIVOS: Determinar la prevalencia de adenomas diagnosticados durante todos los estudios endoscópicos realizados en un periodo de 5 años.

Establecer los factores de riesgo asociados a adenomas colorrectales.

Estratificar en diferentes tipos de riesgo según la historia clínica del paciente y correlacionarlo con los hallazgos anatomopatológicos de los pólipos y el grado de displasia.

PACIENTES Y MÉTODOS: Estudio observacional, retrospectivo, analítico. La población estudiada estuvo conformada por pacientes mayores de 18 años que se realizaron una videocolonoscopía (VCC) en el servicio de Gastroenterología de la Clínica Universitaria Reina Fabiola, en el período comprendido entre 1 diciembre de 2011 y 30 abril de 2017. Las fuentes de datos fueron informes de historia clínica, resultados de estudios endoscópicos y de anatomía patológica.Se analizaron variables demográficas, factores de riesgo para CCR, resultado anatomo-patológico de los pólipos y grado de displasia. Análisis estadístico: Para determinar la relación de diferentes factores de riesgos con el tipo de pólipo obtenido, se realizó un análisis univariado; test de Chi Cuadrado para variables categóricas y test T de Student para variables continuas. Todas las variables significativas en el análisis univariado se incluyeron en un análisis multivariado de regresión logística múltiple.

RESULTADOS: La prevalencia de adenomas fue del 26\%. Se halló una asociación entre el sexo masculino $(\mathrm{p}=0,44)$, edad mayor a 50 años $(\mathrm{p}=0,001)$ y la detección de pólipos durante las colonoscopias de SCCR. Pertenecer a un grupo de riesgo determinado no influyó en el diagnóstico de adenomas $(\mathrm{p}=0,156)$.

CONCLUSIONES: La prevalencia de pólipos de colon fue similar a la reportada por otros estudios.En el programa de SCCR, se observó una mayor frecuencia de pólipos en pacientes de sexo masculino y mayores de 50 años. Los grupos de riesgo no influyeron en el diagnóstico de adenoma.

Palabras claves: Screening de cáncer colorrectal, adenoma colorrectal, factores de riesgo, test de screening, salud pública. 


\begin{abstract}
INTRODUCTION: Colorectal cancer (RCC) is an important health problem worldwide. The goal of screening programs is to make early diagnosis and reduces mortality.

Colonoscopy is a key tool for stratifying the patients and offer timely surveillance.

OBJECTIVES: To determine the prevalence of adenomas diagnosed during all colonoscopy performed over a period of 5 years.

To establish associated risk factors.

To stratify patients risk according to the clinical history and their correlation with the pathological findings. PATIENTS AND METHODS: This is an observational, retrospective, analytical study. Inclusion criteria: patients over 18 years of age who underwent a colonoscopy for a period of 5 years. Demographic variables and anatomo-pathological result of the polyps were analyzed. Statistical analysis: The characteristics of the simple were analyzed with descriptive statistics. To determine the relationship of different risk factors with the type of polyp obtained, a univariate analysis was performed; Chi Square test for categorical variables and Student's T test for continuous variables.

RESULTS: The prevalence of adenomas was $26 \%$. The detection of polyps during SCCR colonoscopies was associated with male sex $(p=0.44)$, andolder than 50 years $(p=0.001)$. Belonging to a specific risk group did not influence the diagnosis of adenomas $(\mathrm{p}=0.156)$.

CONCLUSION: The prevalence of polyps was similar to that reported by other studies. We found an association between demographic factors (male sex and age) and the detection of polyps during an SCCR program.Belonging to a specific risk group did not influence the diagnosis of adenomas.
\end{abstract}

Keywords: Colorectal cancer screening, colorectal adenoma, risk factors, screening tests, public health.

\title{
Introducción
}

El cáncer colorrectal (CCR) representa un problema de salud pública mundial. En Argentina se producen 13500 nuevos casos cada año, de los cuales 7200 son hombres y 6300 son mujeres ${ }^{1}$. Constituye en la actualidad la tercera neoplasia en orden de incidencia en nuestro país en ambos sexos, representando la segunda causa de muerte por cáncer, después del cáncer de pulmón ${ }^{2}$.

La mayoría de los CCR se desarrollan a partir de cambios histopatológicos adquiridos, que determinan la secuencia conocida como adenomacarcinoma, estimándose que se requieren 10 años aproximadamente para que ocurra dicha transición ${ }^{3}$.

El objetivo de la participación de los pacientes en programas de screening de cáncer colorrectal (SCCR) es realizar el diagnostico en estadios tempranos, irrumpir la secuencia carcinogénica y reducir la mortalidad de la enfermedad ${ }^{4,5}$.

En estudios recientes se pudo observar que a partir de la utilización de equipos de alta definición, la tasa de detección de adenomas en los programas de SCCR se presenta en el $40 \%$ al $60 \%$ de los pacientes, mientras que al utilizar endoscopios convencionales la prevalencia de dichos adenomas se reduce entre el 15 y $30 \% \%^{6,7,8}$. Por otra parte a pesar del aumento en los adenomas detectados, la tasa de anual de nuevos cánceres ha declinado entre un 2 a $4 \%$ en el período comprendido entre el año 2002 y $2010^{9}$. Investigaciones previas concluyeron que la remoción de los adenomas reduce la mortalidad por CCR a 10 años, equiparándola con aquellos pacientes sin lesiones en las colonoscopias de screening ${ }^{10}$.

En la patogenia del CCR intervienen factores genéticos y medioambientales, como por ejemplo el tabaquismo, sedentarismo y obesidad entre otros. Para muchos individuos, la edad es el principal factor de riesgo, presentando un aumento en la incidencia a partir de la sexta década de la vida $^{3,12}$. En base a estos datos, la mayoría de las guías recomiendan la realización de una colonoscopia de screening en todo aquel individuo asintomático mayor a 50 años $^{13}$. Pese a esto el CCR se presenta entre el 7 al $9 \%$ en menores de 50 años, y se comporta en este grupo etario de una forma más agresiva ${ }^{13,14}$. Las diferencias en la detección de lesiones de más del doble en hombres que en mujeres se produce en la mayoría de los programas de cribado, por lo que el sexo masculino representa un factor de riesgo adicional $^{15,16}$.

Aunque no todas las influencias genéticas están bien definidas y conocidas $^{17}$, existen factores genéticos que contribuyen al riesgo; los cuales se estima que ocurren en el $30 \%$ de los casos, siendo el Síndrome de Lynch y la Poliposis Adenomatosa Familiar (PAF) ejemplos de ello. Así mismo pacientes con antecedentes personales de CCR, adenomas o enfermedad inflamatoria intestinal (EII) tienen un riesgo más elevado que la población general20. Individuos con historia

Revista Methodo: Investigación Aplicada a las Ciencias Biológicas. Universidad Católica de Córdoba. Jacinto 
familiar de CCR (principalmente en familiares de primer grado) o pólipos adenomatosos tienen mayor riesgo en comparación con aquellos que no presentan antecedentes heredofamiliares ${ }^{11,18}$.

Finalmente, el riesgo de neoplasia avanzada en la colonoscopia de seguimiento depende del número, tamaño y la histología de los adenomas de base así como de la calidad de la exanimación. Los pólipos adenomatosos mayores a $1 \mathrm{~cm}$, con histología vellosa o displasia de alto grado, presentan características que aumentan el riesgo de desarrollar una neoplasia, por lo que el seguimiento por medio de colonoscopía resulta esencial $^{19}$.

\section{Objetivos}

- Determinar la prevalencia de adenomas diagnosticados durante todos los estudios endoscópicos realizados en un periodo de 5 años.

- Establecer los factores de riesgo asociados a adenomas colorrectales.

- Estratificar en diferentes tipos de riesgo según la historia clínica del paciente y correlacionarlo con los hallazgos anatomopatológicos de los pólipos y el grado de displasia.

\section{Materiales y métodos}

Se incluyeron pacientes mayores de 18 años que se realizaron una colonoscopía por el Servicio de Gastroenterología de la Clínica Universitaria Reina Fabiola, durante el período comprendido entre 1 diciembre de 2011 y 30 abril de 2017.

\section{Criterios de exclusión}

Falta de datos a registrar en historia clínica e imposibles de obtener por medio de registros de médicos de cabecera.

\section{Fuentes de datos}

Historia clínica electrónica de los pacientes que se realizaron estudio endoscópico en el período previamente citado.

\section{Variables a registrar}

1) Características demográficas: sexo y edad.

2) Motivo por el cual se solicitó el estudio endoscópico: Se registrará si el paciente se realizó el estudio endoscópico debido a la presencia de síntomas o como screening/vigilancia debido a los antecedentes personales y familiares de pólipos o CCR.
3) Antecedentes heredofamiliares de pólipos o cáncer colorrectal.

4) Estratificación de riesgo según los antecedentes personales y familiares registrados en historia clínica electrónica.

Tabla 1: Clasificación de riesgo según antecedentes de historia clínica ${ }^{1.2}$

\begin{tabular}{|l|l|l|}
\hline \multicolumn{1}{|c|}{ Riesgo bajo } & \multicolumn{1}{c|}{ Riesgo intermedio } & Riesgo elevado \\
\hline Edad $>50$ años & Adenomas previos & $\begin{array}{l}\text { Poliposis } \\
\text { Adenomatosa } \\
\text { Familiar }\end{array}$ \\
\hline $\begin{array}{l}\text { Familiar } 2^{\circ} \circ 3^{\circ} \text { con } \\
\text { antecedentes de } \\
\text { adenoma o } \\
\text { adenocarcinoma }\end{array}$ & $\begin{array}{l}\text { Adenocarcinoma } \\
\text { resecado previamente }\end{array}$ & Sindrome de Lynch \\
\hline $\begin{array}{l}\text { Familiar } 1^{\circ} \text { con } \\
\text { adenocarcinoma }>60 \text { años } \\
\text { o en dos familiares de } \\
\text { segundo grado }\end{array}$ & $\begin{array}{l}\text { Antecedente CCR ० } \\
\text { pólipos adenomatosos } \\
\text { en } 1 \text { familiar de } 1^{\circ} \\
\text { menor a } 60 \text { años } \circ 2 \\
\text { familiares de } 1^{\circ} \text { grado } \\
\text { independientemente } \\
\text { de la edad }\end{array}$ & $\begin{array}{l}\text { Infermedinal Inflamatoria } \\
\text { Intal }\end{array}$ \\
\hline
\end{tabular}

5) Resultado de colonoscopía: Número de pólipos detectados, ubicación (colon derecho, transverso, izquierdo, recto y otros) y tamaño $(<1 \mathrm{~cm}$, entre 1$2 \mathrm{~cm}$ y $>2 \mathrm{~cm}$ ).

6) Resultado de Anatomía patológica: $\mathrm{Se}$ considerará pólipo a todo aquel que presenta características histopatologías de pólipos no adenomatosos o adenomatosos en todas sus variantes.

Se utilizará la clasificación VIENNA para la categorización histológica ${ }^{22,23}$. (Tabla 2)

Tabla 2: Clasificación VIENNA

\begin{tabular}{|l|}
\hline Categoría 1: negativo para displasia \\
Categoría 2: indefinido para displasia \\
Categoría 3: displasia de bajo grado \\
Categoría 4: displasia de alto grado: \\
a-displasia de alto grado \\
b- carcinoma in situ \\
c- sospechoso de carcinoma invasivo \\
Categoria 5: neoplasia invasiva \\
a: adenocarcinoma intramucoso \\
b- adenocarcinoma submucoso
\end{tabular}

\section{Análisis Estadístico}

Las características de la muestra de pacientes se analizaron con estadística descriptiva. Para determinar la relación de diferentes factores de riesgos con el tipo de pólipo obtenido, se realizó un análisis univariado; test de Chi Cuadrado para variables categóricas y test $\mathrm{T}$ de Student para variables continuas. El análisis estadístico se 
realizó utilizando el programa SPSS versión 21 para Windows.

\section{Consideraciones éticas}

Se tuvo en cuenta la confidencialidad de los datos y los recaudos éticos en base a la Declaración de Helsinki (2008), la ley de confidencialidad de datos personales (25.326), el sistema de evaluación, registro y fiscalización de las investigaciones en salud (ley 9694 de Córdoba) y la Guía de Buenas Prácticas Clínicas en Investigación en Salud de la Administración Nacional de Medicamentos, Alimentos y Tecnología Médica (A.N.M.A.T.).

Cabe destacar que esta investigación se realizó con datos de estudios ya obtenidos, donde se constata el consentimiento informado firmado por ambas partes; no se llevarán a cabo estudios adicionales a ningún paciente. Es decir, para la realización del estudio endoscópico, se informó y legalizó dicha práctica por medio de consentimiento informado de ésta institución; donde en el inciso 5, se informa la posibilidad de uso de datos, filmación o fotografía, para fines diagnósticos, docentes o de investigación, siempre y cuando se preserve la identidad de la persona.

\section{Resultados}

De los 1074 pacientes estudiados, 585 correspondían al sexo masculino $(54,5 \%)$ y 489 al femenino $(45,5 \%)$. La edad media (desvío estándar) fue de 53.0 (13.0) años (Figura 1).

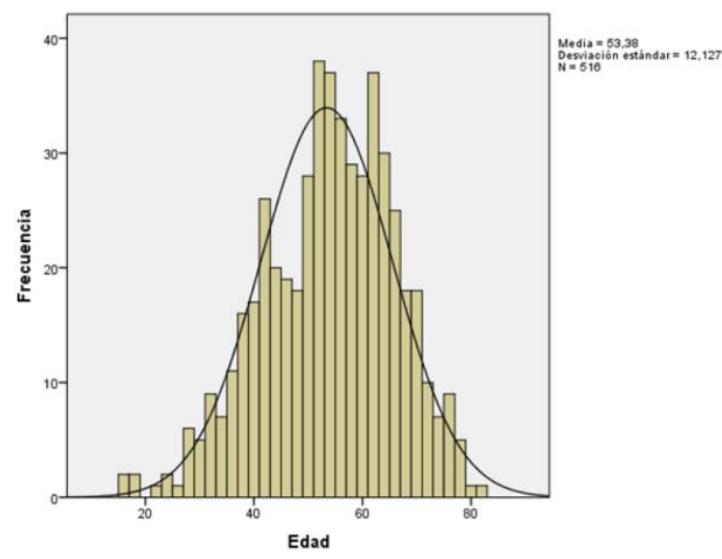

Figura 1: Mediana de edad de todos los pacientes sometidos a una colonoscopía de screening con su correspondiente desvío estándar.

El motivo de solicitud de la colonoscopía para SCCR y seguimiento correspondió al $48 \%$ de la muestra, siendo el $52 \%$ restante debido a síntomas. $\mathrm{Al}$ analizar el total de colonoscopias, en el 26,5\% se detectaron pólipos $(\mathrm{n}=285)$, las cuáles en su mayor porcentaje fueron realizadas para SCCR (54\%). En el 26\% del total de colonoscopias para SCCR se encontraron pólipos. Este grupo de paciente tenía una edad promedio de 57 años. Al considerar el sexo predominó fuertemente el sexo masculino $(53,3 \%$ vs $36,8 \%)$, con una edad promedio de 55 años. La tasa de detección global de pólipos adenomatosos fue del $16 \%$.

Del total de pólipos presentaron en su mayoría pólipos adenomatosos (53,1\%); por orden de frecuencia tubulares $110 \quad(38,6 \%), \quad 30$ tubulovellosos $(10,5 \%) 10$ serratos el $3,5 \%$ y 3 vellosos el $1,1 \%$. Se encontraron132 (46,3\%) pólipos hiperplásicos.

Al tener en cuenta la ubicación las lesiones en su mayor porcentaje se encontraron en colon transverso (35\%) y colon izquierdo (28\%), seguidos del colon derecho $(13,33 \%)$, recto $(11 \%)$ y ciego (11\%).

Con respecto al tamaño, en el $82,80 \%$ se presentaron lesiones menores a $1 \mathrm{~cm}$, siendo en su mayor porcentaje de histología hiperplásica, con una clasificación histológica de Vienna Paris de 1 $(n=127)$ seguido de los tubulares $(n=97)$. Dos lesiones menores a $1 \mathrm{~cm}$, constituyeron adenocarcinomas. Los pólipos entre 1 y $2 \mathrm{~cm}$ correspondieron a tan solo el $8,42 \%$ del total de las lesiones, presentando en su mayoría histología tubulovellosa (12), seguidos de 8 tubulares, 3 hiperplásicos, 1 serrato. Veinticinco $(8 \%)$ de los pólipos fueron mayores a $2 \mathrm{~cm}$, predominando la histología tubulovellosa. A mayor tamaño del pólipo, se observó una peor histología, siendo esta relación estadísticamente significativa $(\mathrm{p}=0,001)$ (Tabla 3).

Tabla 3: Características histológicas de los pólipos encontrados en todas las colonoscopías durante un período de 5 años y la relación con el tamaño del mismo.

\begin{tabular}{|c|c|c|c|c|}
\hline \multirow{2}{*}{$\begin{array}{l}\text { Subtipo } \\
\text { histológico }\end{array}$} & \multicolumn{3}{|c|}{ Tamaño de pólipo } & \multirow{2}{*}{$\frac{\text { Valor }}{\text { dep }}$} \\
\hline & $\leq 1 \mathrm{~cm}$ & $>1 \mathrm{~cm}<2 \mathrm{~cm}$ & $\geq 2 \mathrm{~cm}$ & \\
\hline Hiperplásico & $127(96,2 \%)$ & $3(2,3 \%)$ & $2(1,5 \%)$ & 0,001 \\
\hline Serrato & $7(70 \%)$ & $1(10 \%)$ & $2(20 \%)$ & \\
\hline Tubular & $97(88,2 \%)$ & $8(7,3 \%)$ & $5(4,5 \%)$ & \\
\hline Velloso & $0(0 \%)$ & $0(0 \%)$ & $3(100 \%)$ & \\
\hline Tubulovelloso & $5(16,7 \%)$ & $12(40 \%)$ & $13(43,3 \%)$ & \\
\hline
\end{tabular}

Al clasificar a los pacientes de SCCR de acuerdo al riesgo en la historia clínica (Tabla 3), su mayor porcentaje perteneció al grupo de riesgo intermedio $(\mathrm{n}=252,48,8 \%)$ seguido del bajo $(\mathrm{n}=228,44,2 \%)$ y en último lugar al elevado $(\mathrm{n}=36,7 \%)$.

En los pacientes con riesgo bajo, 66 (43\%) presentaron pólipos; en el grupo de riesgo intermedio, $81(53 \%)$ presentó pólipos y en el grupo de riesgo elevado se encontraron pólipos en $6(4 \%)$ pacientes. (Tabla 4) No se encontró una asociación estadísticamente significativa en pertenecer a un grupo de riesgo determinado y la

Revista Methodo: Investigación Aplicada a las Ciencias Biológicas. Universidad Católica de Córdoba. Jacinto Ríos 571 Bo Gral. Paz. X5004FXS. Córdoba. Argentina. Tel.: (54) 3514517299 / Correo: methodo@ucc.edu.ar 
presencia o ausencia de pólipos durante las colonoscopias realizadas para SCCR $(\mathrm{p}=0,156)$.

Tabla 4: Relación entre el sexo masculino y los diferentes grupos de riesgo, en base a la presencia o ausencia de pólipos.

\begin{tabular}{|c|c|c|c|c|c|}
\hline & \multicolumn{3}{|c|}{ Estratificación de riesgo por SCCR } & \multirow{2}{*}{$\frac{\text { Valor }}{\mathrm{p}}$} \\
\hline & & Bajo & Intermedio & Elevado & \\
\hline \multirow[t]{2}{*}{$\underline{\text { Sex0 }}$} & Masculino & $127(46,7 \%)$ & $128(47,1 \%)$ & $17(16,3 \%)$ & \multirow[t]{2}{*}{0,44} \\
\hline & Femenino & $101(41,4 \%)$ & $124(50,8 \%)$ & $19(7,8 \%)$ & \\
\hline \multirow[t]{2}{*}{ Pólipos } & Presencia & $66(43,1 \%)$ & $81(52,9 \%)$ & $6(3,9 \%)$ & \multirow[t]{2}{*}{1,56} \\
\hline & Ausencia & $162(44,6 \%)$ & $171(47,1 \%)$ & $30(8,3 \%)$ & \\
\hline
\end{tabular}

La distribución del sexo de acuerdo al riesgo fue similar en todos los grupos $(\mathrm{p}=0,44)$. A pesar de ello, existió una asociación estadísticamente significativa entre la detección de pólipos durante el SCCR y el sexo masculino ( $\mathrm{p}=0,017)$.

La media de edad en el SCCR para los pacientes con pólipos fue de 56 años vs 52, en aquellos sin lesiones $(\mathrm{p}=0.010)($ Tabla 5).

Tabla 5: Relación entre el sexo y la edad mayor a 50 años con la presencia de pólipos.

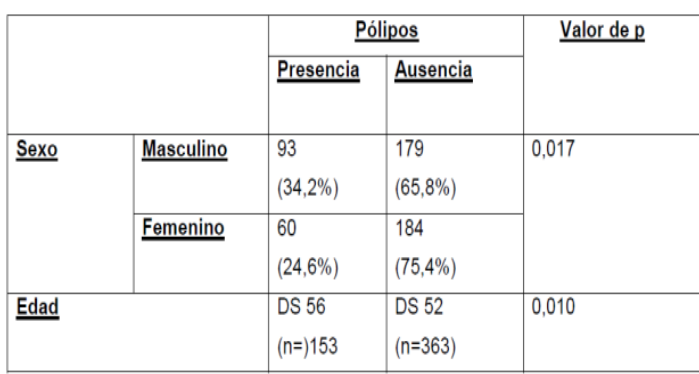

\section{Discusión}

El cáncer colorrectal (CCR) es una de las neoplasias más frecuentes en los países occidentales. En Argentina es el segundo de mayor mortalidad en ambos sexos con más de 7.000 fallecimientos anuales ${ }^{1,2,23}$. Es una enfermedad que se beneficia de programas de prevención en pacientes de riesgo promedio o elevado, ya que los mismos permiten reducir su incidencia $\mathrm{y}$ mortalidad.

La colonoscopía representa uno de los test disponibles para realizar en pacientes con síntomas o que se incluyan dentro de un programa de screening de cáncer colorrectal (SCCR), permitiendo al mismo tiempo un enfoque diagnóstico y terapéutico ${ }^{4}$.

En nuestro grupo de estudio, el promedio de edad fue similar al analizado en otros grupos (53 años con SD de 13 años) de screening. Las últimas evidencias sugieren que la incidencia de CCR está aumentando en adultos menores a 50 años hasta en un $51 \%$, representando del 7-15\% del total de los $\mathrm{CCR}^{13}$. En base a esta evidencia la mayoría de las guías actuales sugieren iniciar el SCCR a los 45 años en personas de riesgo promedio, y se espera que las próximas muestras de pacientes estén constituidas por individuos más jóvenes ${ }^{23,24}$.

No se encontró una asociación significativa en pertenecer a un grupo de riesgo y la ausencia o presencia de pólipos. La organización de los pacientes en diferentes grupos de riesgo es útil para poder realizar los controles de una manera oportuna y permitir el diagnóstico de CCR en estadios tempranos. La ausencia de asociación puede verse influenciada por un tamaño muestral pequeño, acortamiento en el tiempo de los controles y diversas variables que no han sido estudiadas y que influyen a la hora de determinar la tasa de detección de adenomas (ej. preparación de colon, llegada a ciego, tiempo de retirada, tasa de detección de pólipos etc.).

La mediana de edad de los pacientes con lesiones fue ligeramente superior a aquellos pacientes que no presentaron lesiones, presentando esta asociación una relación estadísticamente significativa. La edad avanzada representa un factor de riesgo ampliamente conocido de lesiones adenomatosas en los grupos de SCCR, determinando que el tiempo influye en el desarrollo de lesiones pre-neoplásicas y neoplásicas ${ }^{25}$.

Pertenecer al sexo masculino en nuestra cohorte es un factor de riesgo con asociación estadísticamente significativa para presentar pólipos. El rol del sexo estaría determinado por el efecto promotor de la testosterona, el cual podría explicar el mayor riesgo en el desarrollo de adenomas colorrectales $^{27-29}$.

Existen otros factores de riesgo independientes que influyen en la detección de adenomas como el antecedente de hipertensión arterial, diabetes, sedentarismo, índice de masa corporal elevado (IMC), consumo de tabaco y alcohol, dietas con ingesta elevada de carnes rojas y procesadas ${ }^{24,30}$. Estos no han sido considerados en la población de estudio debido a que al ser un análisis retrospectivo no se contó con estos datos dentro de la historia clínica. Podrían ser una herramienta interesante a tener en cuenta en futuros programa de screening. Los adenomas se encontraron principalmente en el lado izquierdo y en menor medida en otras localizaciones como colon derecho y recto. Estos datos coinciden con la prevalencia elevada de CCR del lado izquierdo y son similares en otros grupos de $\mathrm{SCCR}^{31}$. Esta situación se da debido a diversas razones entra las que cabe destacar la mayor dificultad en alcanzar la correcta limpieza y visualización del colon ascendente, y la mayor incidencia de adenomas planos o lesiones de 
crecimiento lateral en esta localización, lo que hace que su diagnóstico sea más difícil ${ }^{17,33,34}$.

El subtipo histológico más predominante fueron los pólipos adenomatosos, que constituyeron en total un $53,1 \%$ de la muestra. Dentro de estos, se encontraron por orden frecuencia los tubulares $(38,6 \%)$, tubulovellosos $(10,5 \%)$, serratos $(3,5 \%)$ y vellosos $(1,1 \%)$. El $46,3 \%$ restante fueron pólipos hiperplásicos. Todos los pólipos adenomatosos pueden progresar a carcinoma. El riesgo de malignidad de los mismos está determinado por la arquitectura histológica, el tamaño y el grado de displasia. En esta muestra se comprobó la asociación entre el tamaño del pólipo y el grado o subtipo histológico: a mayor tamaño, peor histología. Esta asociación ha sido corroborada en muchos estudios previos. Lesiones mayores a $20 \mathrm{~mm}$, con displasia de alto grado e histología vellosa son criterios de adenomas $\operatorname{avanzados}^{35}$. Por otra parte, el tamaño de las lesiones influye en la probabilidad de su visualización. Sin embargo, en nuestra muestra el mayor porcentaje de lesiones observadas fueron menores a $1 \mathrm{~cm}$ y correspondieron a pólipos hiperplásicos.

Se encontraron dos lesiones menores a $1 \mathrm{~cm}$ que cuyo informe anatomo patológico se correspondió con adenocarcinomas. A partir de estos resultados se plantea el interrogante de la naturaleza de los pólipos diminutos (1-5 $\mathrm{mm}$ ) y pequeños $(6-9 \mathrm{~mm})$. Vleugels et al, en una revisión sistemática estudiaron la historia natural de los mismos y pudieron estimar que la tasa de progresión a adenomas avanzados o CCR es muy bajaz6. Muchos autores plantean que la remoción puede ser más lesiva que beneficiosa, debido que aumenta el riesgo de eventos adversos, exámenes histológicos y la vigilancia endoscópica comparada con los beneficios mínimos de protección contra el CCR. Se espera que la aplicación de nuevas tecnologías endoscópicas como la cromoendoscopia y utilización de endoscopia de alta resolución, ayuden en la aproximación histológica permitiendo mejorar la conducta terapéutica en este tipo de lesiones ${ }^{40}$.

La tasa de detección de adenoma (proporción de colonoscopías de screening en población promedio en las que se detectó al menos un adenoma) se correlaciona inversamente con el CCR de intervalo y con una disminución de su mortalidad ${ }^{41}$.

A pesar de que el estudio no fue diseñado para valorar exclusivamente las colonoscopías de SCCR y su calidad, los pólipos detectados en el total de colonoscopías fue del $16 \%$; siendo este valor inferior al reportado por otros estudios. En grandes estudios multicéntricos de Italia, la tasa media de detección de pólipos fue del $35 \%$. En poblaciones occidentales, la tasa de detección de adenomas (TDA) en las colonoscopías de screening para individuos mayores de 50 es del $25 \%$ para hombres y del $15 \%$ para mujeres. Actualmente la ASGE recomienda una TDA de $30 \%$ en hombres y del $20 \%$ en mujeres estableciendo este punto de cohorte ya que estos valores se correlacionan con un bajo riesgo de cáncer de intervalo ${ }^{41}$.

Dentro de los factores de impacto en la TDA se pueden enumerar: preparación colónica satisfactoria, tiempo de retirada mayor a 6 minutos, una correcta visualización del colon derecho e intervención de un segundo observador durante el procedimiento ${ }^{41}$.

Cabe recalcar que las colonoscopías realizadas en este período de tiempo fueron por medio de endoscopios baja definición lo que influye directamente en la visualización de lesiones. Estudios previos retrospectivos y prospectivos han determinado que la prevalencia de adenomas es del 15 al $30 \%$ en endoscopias de baja resolución, y que al utilizar de endoscopios de alta resolución se eleva hasta el $50 \%$ por lo que actualmente la ESGE recomienda utilizar endoscopios de alta calidad para la colonoscopia de screening en pacientes de riesgo normal ${ }^{6-8}$.

De la misma manera la aplicación de cromo endoscopia virtual aumenta la TDA, permite una mejor visualización de lesiones planas o del lado derecho en comparación con la colonoscopia convencional $^{37}$

La aplicación de endoscopios de mayor ángulo de visualización no presenta diferencias en el número de adenomas detectados, sino que permite una reducción en el tiempo de retirada del $15 \%$, pero no ha mostrado reducir o eliminar la tasa de adenomas perdidos ${ }^{37-41}$.

Dentro de las limitaciones del estudio se encuentra que en primer lugar es un estudio retrospectivo en donde los datos fueron obtenidos a partir de la historia clínica electrónica, lo que podría generar limitaciones en la obtención de la información (sesgo de memoria de la historia clínica) y en la recolección de otras variables demográficas que influyen en la prevalencia de adenomas tales como tabaquismo, alcoholismo, obesidad, diabetes, uso de aspirina, presencia de cirugía abdominal y enfermedad diverticular.

Por otra parte, los estudios endoscópicos fueron realizados por diversos operadores sin estudios previos de correlación interobservador (sesgo de información) y en ausencia de técnicas de magnificación o de cromoendoscopia que refuerzan y agudizan la detección macroscópica, como ya se mencionó previamente.

A pesar de esto, es un estudio que aporta luz respecto a la prevalencia de adenomas en los pacientes sometidos a una colonoscopía dentro del servicio, las características histológicas y su ubicación predominante. 
Sería interesante realizar futuros estudios en programas de screening considerando los factores determinantes en la calidad de endoscopía y la utilización de nuevas tecnologías adquiridas para determinar su influencia en la detección de adenomas.

Resulta de vital importancia monitorizar la calidad de las endoscopías de screening para poder brindar una herramienta diagnóstica precoz en el manejo de los pacientes.

\section{Conclusiones}

La prevalencia de pólipos fue similar a la reportada por otros estudios. Existe una asociación entre el sexo masculino y edad mayor a 50 años, en la detección de pólipos durante las colonoscopias realizadas en un programa de SCCR. Las lesiones encontradas empeoran su histología, conforme aumenta el tamaño de la misma. Pertenecer a un grupo de riesgo determinado, no influye en la presencia o ausencia de pólipos durante las colonoscopias realizadas para SCCR, pero es útil para realizar el seguimiento oportuno adecuado.

\section{Bibliografía}

1. Instituto Nacional del Cáncer [Internet]. Argentina: año 2015. Ministerio de Salud de la Nación. Programa Nacional de Detección y Prevención Temprana del Cáncer Colorrectal. Instituto. Disponible en http://www.msal.gov.ar/inc/el-inc-y-susprogramas/programa-nacional-deprevencion-y-deteccion-del-cancercolorrectal/

2. Instituto Nacional del Cáncer [Internet]. Argentina: año 2016. Ministerio de Salud de la Nación. Vigilancia epidemiológica del Cáncer. Análisis de situación de salud por Cáncer. Argentina, 2016. Disponible en http://www.msal.gob.ar/images/stories/bes/gr aficos/0000000925cnt-2016-12-21-boletinepidemiologia.pdf

3. Park Y, Kim HS, Park JJ et al. A Simple Scoring ModelforAdvancedColorectal Neoplasm in Asymptomatic Subjects Aged 40 - 49 Years. BMC Gastroenterology, 2017; (2017)17:7.

4. Young GP, Senore C, Mandel JS et al.Recommendationsfor a StepWiseComparativeApproachtotheEvaluation of New ScreeningTestsforColorectalCancer. Cancer. 2016; 122 (6): 826-839.

5. García-Albéniz X, Bretthauer M. \&Hernán M.A. Effectiveness. Screening
Colonoscopyto Prevent Colorectal Cancer Among Medicare BeneficiariesAged 70 to 79 Years. Tothe Editor: García-Albéniz and colleagues. Annals of Internal Medicine. American College of Physicians. 2017; 166 (10): 18-26.

6. Anderson JC, Baron JA, Ahnen DJ et al. Factors Associated With Shorter Colonoscopy Surveillance Intervals for Patients With LowRisk Colorectal Adenomas and Effects on Outcome. Gastroenterology. 2017; 152(8): 1933-1943.

7. Anderson JC, Stein B, Kahi CJ et al. Association of Smoking and flat adenomas: Resultsfroman Asymptomatic Population Screened with a High-definitionColonoscope. Gastrointestinal Endoscopy. 2010; 71 (7):1234-1240.

8. Dekker E, Dinis-ribeiro M, Ferlitsch M et al. Post-polypectomy Colonoscopy Surveillance: EuropeanSociety of Gastrointestinal Endoscopy (ESGE) Guideline. 2013; 45 (10): 842-851.

9. Richardson LC, Tai M, Rim SH et al. Vital signs: Colorectal Cancer Screening, Incidence, and Mortality-UnitedStates, 20022010. Centers forDisease Control and Prevention (CDC). 2011;60(26):884-9.

10. Zauber AG., Winawer SJ, O'Brien $\mathrm{M}$ et al. ColonoscopicPolypectomy and LongTermPrevention of Colorectal-CancerDeaths. New England Journal: 2012. 366(8): 687-696.

11. Winawer S, Zauber A, Gerdes H Et al. Risk of ColorectalCancer in the Families of Patientswith Adenomatouspolyps.The New England Journal of Medicine. 1996; 334 (2): 82-87.

12. Goldvaser H, Shroitman NK, Ben-aharon I et al. Octogenarianpatientswithcolorectalcancer: Characterizinganemergingclinicalentity.

WorldJournal of Gastroenterology. 2017; 23(8), 1387-1396.

13. Fairley TL, Cardinez CJ, Martin J, et al. Colorectalcancer in US adultsyoungerthan 50 years of age, 1998-2001. Cancer. 2006; 107(5):1153-61.

14. Kim JY, Jung YS, Park JH et al. Differentriskfactorsforadvancedcolorectalneo plasm in youngadults. World Journal of Gastroenterology. 2016; 22(13), 3611-3620.

15. Qureshi A, Shihi S, Ali Z, \&Shalaby A. A retrospectivestudy of clinicopathologicalcharacteristics of colonicpolyps 
in adultsseen at a tertiarycare centre. Journal of Pakistan Medical Association. 2017; 67 (1): 12-14.

16. Oluyemi A, Awolola N, OyedejiO.Clinicopathologicreview of polypsbiopsied at colonoscopy in Lagos, Nigeria. Pan African Medical Journal. 2016; 24 (33): 1-6.

17. Da Silva FC, Wernhoff $P$ \& DominguezBarrera C. Up date on Hereditary Colo-rectal Cancer. AnticancerResarch. 2016; 36(9):4399-406.

18. Samadder NJ, Curtin K, Tuohy TM et al. Risk of colorectalcancer and adenomas inthefamilies of patientswith adenomas: a population-based study in Utah. Gastroenterology.2014; 120(1):35-42.

19. Atkin W, Wooldrage K, Brenner A et al. Adenoma Surveillance and Colorectal CancerIncidence: a retrospective, multicentre, cohortstudy. LancetOncology. 2017; 2045(17), 1-12.

20. Eaden JA, Abrams KR, \& Mayberry JF. Therisk of colorectalcancer in ulcerative colitis: ametanalisis. Gut. 2001; 48 (4): 526535.

21. Schlemper RJ, Riddell RH, Kato Y, et al. The Vienna classification of gastrointestinal epithelial neoplasia. Gut 2000;47 (2):251255.

22. Rubio A, Nesi G, Messerini L et al. The Vienna classification applied to colorectal adenomas. Journal of Gastroenterology and Hepatology. 2006; 21 (2006): 1697-1703.

23. Cubiella J, Marzo Castillejo M et al. Clinical practice guideline. Diagnosis and prevention of colorectal cancer. 2018 Update. GastroenterolHepatol. 2018 Nov;41(9):585596.

24. Wolf AMD, Fontham ETH et al. Colorectal cancer screening for average-risk adults:2018 guideline uptodate from the American Cancer Society. CA Cancer J Clin. 2018 Jul;68(4):250-281.

25. Chen FW, Sundaram V Et al. Advanced-Stage colorectal cancer in persons younger than 50 years not associated with longer duration of symptoms or time to diagnosis. ClinGastroenterolHepatol. 2017 May;15(5):728-737.

26. Brenner H, Hoffmeister M Et al. Risk of progression of advanced adenomas to colorectal cancer by age and sex: estimates based on 840149 screening colonoscopies. Gut. 2007 Nov;56(11):1585-9.

27. AlmadiMa, Alharbi $\mathrm{O}$ et al.Prevalence and characteristics of colonic polyps and adenomas in 2654 colonoscopies in Saudi Arabia.Saudi J Gastroenterol. 2014 MayJun;20(3):154-61.

28. Regula J, Rupinski et al.Colonoscopy in colorectal-cancer screening for detection of advanced neoplasia.NEngl J Med. 2006 Nov 2;355(18):1863-72.

29. Lee Se, Jo HB et al. Characteristics of and risk factors for colorectal neoplasms in Young adults in a screening population. World $\mathbf{J}$ Gastroenterol. 2016 Mar 14;22(10):2981-92.

30. Cahn AT etGiovannucci EL. Primary prevention of colorectal cancer. Gastroenterology. 2010 Jun;138(6):20292043.

31. Aswakul P, Prachayakul V et al. Screening colonoscopy from a large single center of Thailand- something needs to be changed?Asian Pac J Cancer Prev. 2012;13(4):1361-4.

32. Borof ES, Gurudu SR et al. Polyp and adenoma detection rates in the proximal and distal colon. Am J Gastroenterol. 2013 Jun;108(6):993-9.

33. Pace D, Borgaonkar $\mathrm{M}$ et al. Effect of colonoscopy volum on quality indicators. Can J Gastroenterol Hepatol. 2016; 2016:2580894.

34. Bretagne JF, Hamonic $S$ et al. Interendoscopist variability in proximal colon polyp detection is twice higher for serrated polyps than adenomas. World J Gastroenterol. 2016 Oct 14;22(38):8549-8557.

35. Rex DK. Maximizing detection of adenomas and cancers during colonoscopy. Am J Gastroenterol. 2006 Dec;101(12):2866-77.

36. Vleugels JLA, Hazewinkel Y et al. Natural history of diminutive and smallcolorectal polyps: a systematic literature review. GastrointestEndosc. 2017 Jun;85(6):11691176.e1.

37. Aranda-Hernandez J, Hwang J etKandel G. Seeing better evidence based recommendations on optimizing colonoscopy adenoma detection rate. World $\mathbf{J}$ Gastroenterol. 2016 Feb 7;22(5):1767-78.

38. Hoff G, FoersterA et al. Epidemiology of polyps in the rectum and colon. Recovery and evaluation of unresected polyps 2 years after 
detection. Scand J Gastroenterol. 1986 Sep;21(7):853-62.

39. Ueyama T, Kawamoto K et al. Natural history of minute sessile colonic adenomas based on radiographic findings. Is endoscopic removal of every colonic adenoma necessary? Dis Colon Rectum. 1995 Mar;38(3):268-72.

40. Mizuno K, Suzuki Y et al. Natural history of diminutive colorectal polyps: long-term prospective observation by colonoscopy. DigEndosc. 2014 Apr;26Suppl 2:84-9.

41. Douglas K. Rex, Philip S. Schoenfeld et al. Quality indicators for colonoscopy. Gastrointestinal Endoscopy. 2015 Jan; 81 (1):31-53.

42. ShuakatA, Gravely AA et al. Rates of Detection of Adenoma, Sessile Serrated Adenoma, and Advanced Adenoma Are Stable Over Time and Modifiable. Gastroenterology. 2019 Feb;156(3):816-817

\section{(c) (1)(2)(2) \\ BY NC SA}

Revista Methodo: Investigación Aplicada a las Ciencias Biológicas. Universidad Católica de Córdoba. Jacinto Ríos 571 Bo Gral. Paz. X5004FXS. Córdoba. Argentina. Tel.: (54) 3514517299 / Correo: methodo@ucc.edu.ar / Web: methodo.ucc.edu.ar | ARTICULO ORIGINAL Methodo 2019;4(4):139-147 